\title{
Diagnóstico precoz del ductus persistente en recién nacidos de pretérmino con ecocardiografía de contraste
}

\author{
Dr. Ignacio Hernández N.1; Dra. Odette Farń A. 1; Dra. Mafalda Rizzardini P.2 \\ Echocardjographic identification of patent ductus arteriosus
}

\begin{abstract}
Contrast echocardiography (CE) was carried out in 16 newborns less than 72 hours of life weighing under $2.000 \mathrm{~g}$, by inyecting $5 \%$ dextrose in water through an umbilical artery catheter, with simultaneus echocardiographic register of the pulmonary artery (PA). An $X$ ray contrast aortography was made afterwards. According to the intensity of the echos obtained from PA, CE was classified in grade I (weak echos) and grade II (dense echos). The size of PDA as seen in the aortography was classified depending on the ratio of to descending aortic diameter: ratios greater than 0.5 indicated large ductus, ratías less than 0.5 indicated small ductus. The aortographic-echocardiographic correlation was excelent: $1 \mathrm{l}$ cases with aortographic PDA had positive CE: 8 aortographic large PDA with CE grade Il and 3 aortographic small PDA with CE grade I. This CE technique is easy to obtain and credible to detect PDA in prematures, allowing an early medical or surgical treatment.
\end{abstract}

(Key words: Ductus arteriosus, echocardiography, X Ray contrast aortography).

Es un hecho conocido que la incidencia del ductus persistente (DP) en el recién nacido es mayor mientras menor es la edad gestacional. E1 importante desarrollo que ha tenido la neonatología durante las dos últimas décadas determinó la creación de las unidades de cuidados intensivos neonatales, especialmente dotadas con técnicas de ventilación mecánica destinadas al manejo del sindrome de dificultad respiratoria tan frecuente en el prematuro. Ello ha permitido la sobrevida de niños cada vez más inmaduros con el consiguiente aumento de la incidencia del DP. La detección precoz de éste es muy importante ya que un corto-circuito de izquierda a derecha a nivel ductal afecta muy adversamente el estado cardiopulmonar de estos prematuros $5,6,7,13$, 17, 18, 21. En muchos casos los elementos clínicos como soplo cardíaco y pulsos saltones están ausentes y la evaluación con electrocardiograma, radiografía de tórax, no son concluyen$\operatorname{tes}^{13}, 16,17,21$. Las mediciones ecocardiográficas (Modo M) de las dimensiones cardiacas, de la fracción de acortamiento del ventrículo izquie ro y de los intervalos sistólicos, si bien son útiles para sugerir el diagnóstico, tienen importantes limitaciones $e$ inespecificidad $2,3,4,8,10$, 12. $14,15,20$.

El objeto de este trabajo fue evaluar la eco-

1. Cardiologia, hospitai Roberto del Rio.

2. Neonatología, hospital Roberto del Río. cardiografía de contraste (EC) como un método simple e inocuo para diagnosticar en forma precoz el DP en niños de pretémino.

\section{MATERIAL Y METODO}

Se estudiaron 16 recién nacidos de pretérmino hospitalizados en la Unidad de Neonatologial del Hospital Roberto del Río. Se seleccionaron nijos menores de 72 horas de vida cuyo peso eta inferior a 2.000 gramos y sólo aquellos que necesitaron cateterización de arteria umblical cono parte de las téenicas empleadas en el cundado rutinario de estos pacientes. Para efectos del examen se requirió que dicho catéter estuviera ubicado entre la $4^{\text {a }}$ y $8^{\text {a }}$ vértebra dorsal. La presencia de ductus se comprobó mediante aor tografía realizada a través del catéter arterial umbilical usando Hypaque al $60 \%$ diluido al medio con agua destilada en dosis de 1,2 m1 por kilo ds peso. La inyección se efectuó manualmente, tomando placa radiográfica simultánea con el niño en decúbito lateral derecho4, 9. 11, 21.

La magnitud del ductus se catalogó según la relación diámetro menor đuctal/diámetro aorta descendente. Consideramos como ductus pequễo una relación menor de $0.5(+)$ y ductus amplio aquellos con una relación mayor de $0.5(++)$.

Inmediatamente después de realizada la aortografía se efectuó ecocardiograma de contraste. Este se obtuvo inyectando en forma manual por el catéter arterial umbilical $1 \mathrm{ml}$ de suero glucosado al $5 \%$ mientras se visualizaba e inscribía en el ecocardiógrafo la arteria pulmonar (Fig. 1). Fue seleccionada la menor ganancia posible requerida para visualizar claramente la válvula pulmonar. Fn presencia de DP, las microburbujas generadas durante la inyección se transmitieron por el ductus alcanzando la arteria pulmonar a nivel de la válvu. $1 \mathrm{a} 1,19,22$. 
Los ecos resultantes aparecen en el ecocardiograma obscureciendo la vátvula pulmonar en diástole (Fig. 1). Según la intensidad de ecos producidos en el registro, el EC se clasifícó en Grado I' ( + ) si la cantidad de ecos fue escasa y Grado II ( ++ ) si los ecos fueron densos).

Se realzó una evaluación clínica diaria de los niños, con examen físico, radiografía de tórax, electrocardiograma y gases arteriales, además del resto de exámenes empleados en el manejo habitual de estos pacientes.

Los resultados aortográficos y ecocardiográficos se correlacionaron entre sí y con los hallazgos clínicos.

\section{RESULTADOS}

La edad promedio de los nifros estudiados fue de 29,7 horas (rango: 12 a 48 horas). El peso promedio fue 1.258 gramos (rango: 940 a 2.000 g). 10 fueron de sexo femenino, 6 masculino.

La aortografía (Tabla 1) demostró DP en 11 casos: 8 con ductus amplio, 3 pequeño. En 5 fue negativa. Los 8 niños con DP amplio presentaron EC Grado IJ (Fig. 2). Los 3 casos con DP pequeño tenían EC Grado [ (Fig. 3). De los 5 casos con aortografía negativa para DP, 3 tenian EC tarnbién negativo y 2 EC Grado I.
Tabla 1.

\begin{tabular}{cccc}
\hline Casos & Ao & E.C. & Relación AI/Ao \\
\hline 1 & ++ & ++ & 1.6 \\
2 & ++ & ++ & 1.5 \\
3 & ++ & ++ & 1.4 \\
4 & ++ & ++ & 1.4 \\
5 & ++ & ++ & 1.3 \\
6 & ++ & ++ & 1.3 \\
7 & ++ & ++ & 1.2 \\
8 & ++ & ++ & 1.2 \\
9 & + & + & 1.0 \\
10 & + & + & 1.1 \\
11 & + & + & 1.0 \\
12 & - & + & 0.9 \\
13 & - & + & 0.8 \\
14 & - & - & 0.9 \\
15 & - & - & 0.85 \\
16 & - & - & 0.8
\end{tabular}

En el ecocardiograma Modo-M, la relación A]/AO era 1,2 o mayor en $\operatorname{los} 8$ casos con ductus amplio. El resto de los niños presentó una rela. ción AI/AO normal (menor de 1,1).

Al momento del estudjo angio-ecocardiográfico, sólo 1 de los niños (con DP amplio) presentaba soplo cardíaco. El resto no tenía

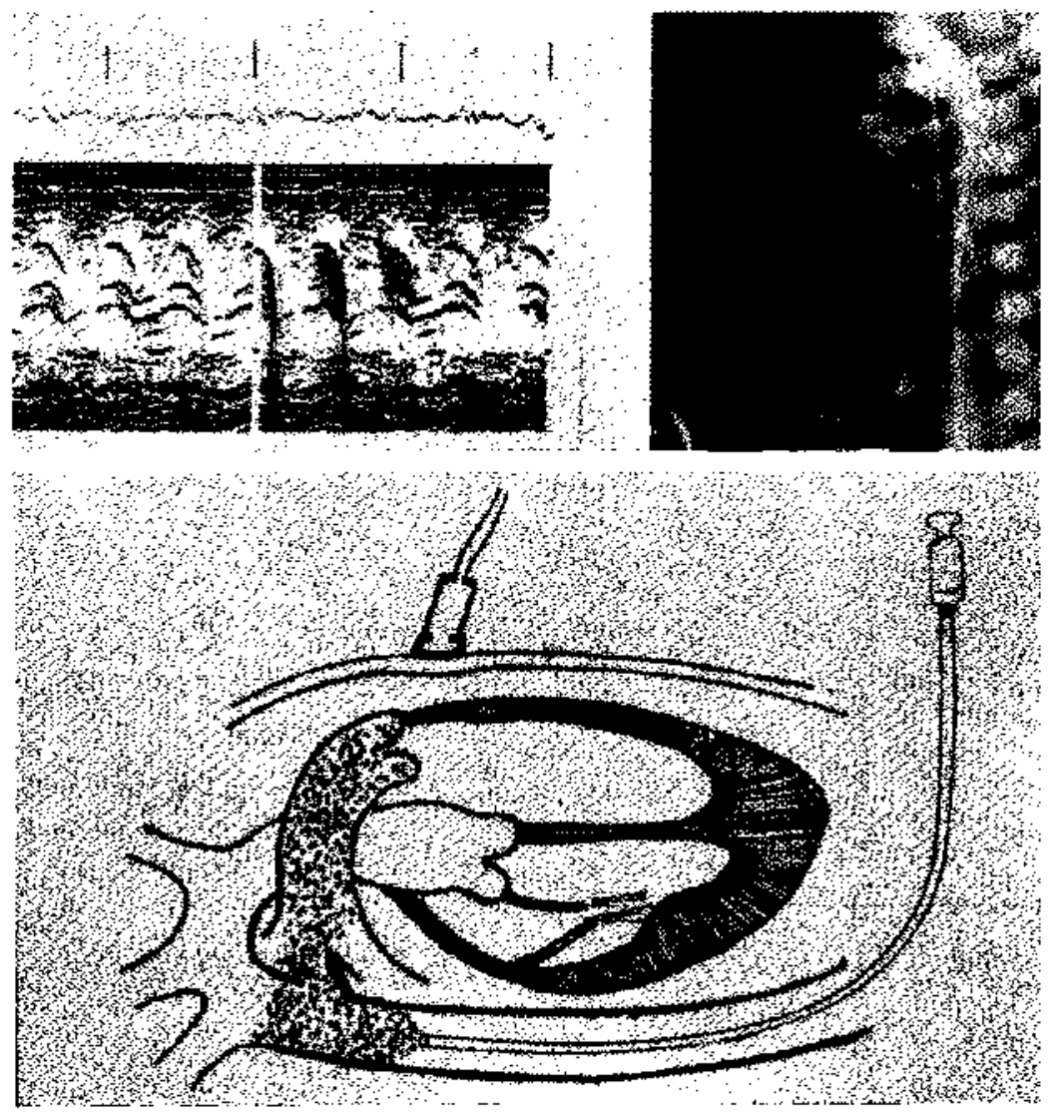

Figura 1. Eccocardjografía de contraste. Registro de arteria pulmonar; la turbulencia creada por la inyección de sueio en arteria aorta se transmite por el ductus a arteria pulmonar. Arriba izquierda: el plano valvular pulmonar muestra una luvia de ecos en diástole. Arriba derecha: confirmación angiográfica del ductus persistente, en el mismo paciente. 

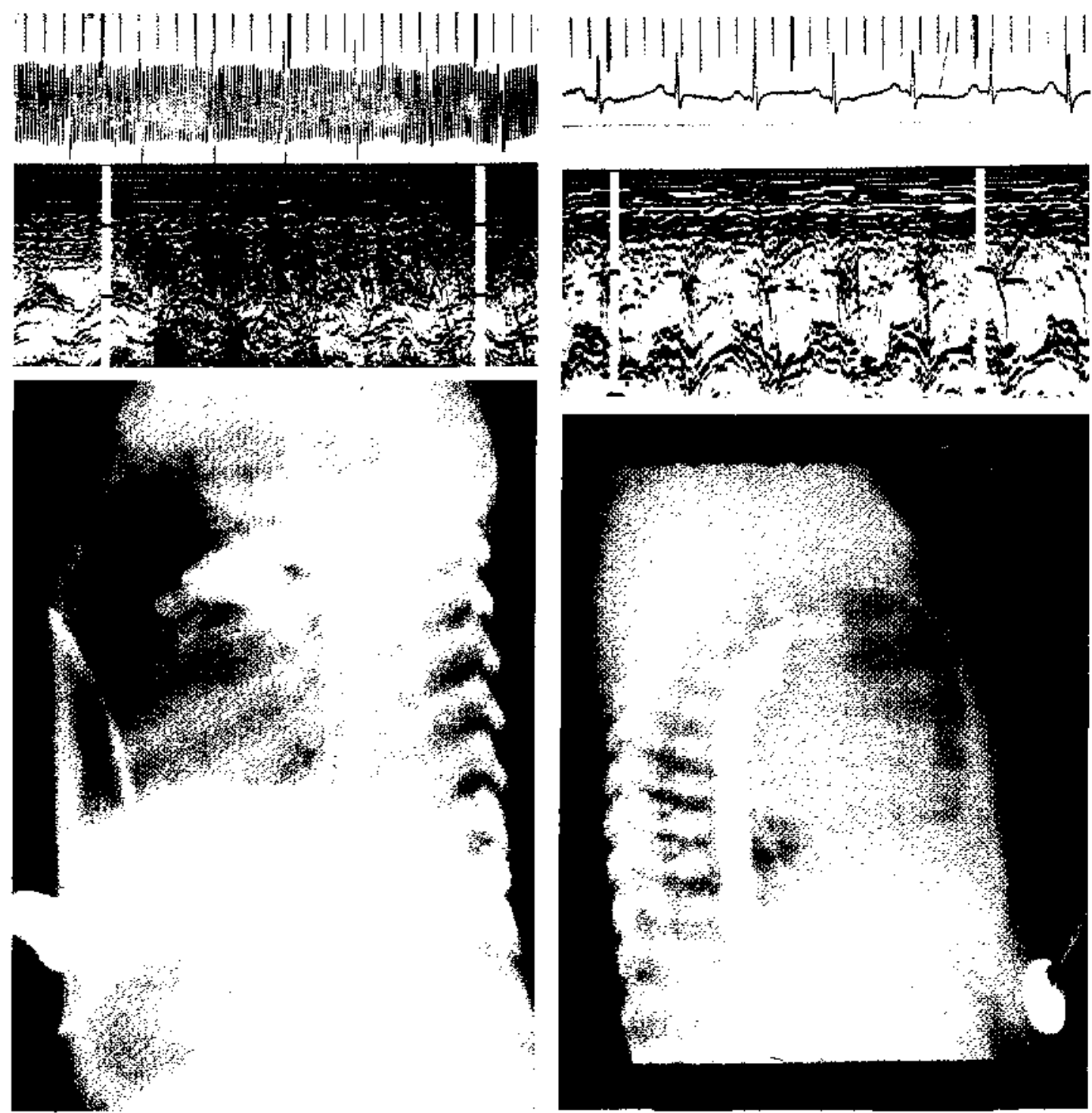

Figura 2. locoutdicerafía de contraste grado ll (arribid) y demostracicin aortograliea de un ductus amplio (abajo). Ef tal thistono niño.

soplos y a ECG y radiografía de torax no presentaban altesaciones significativas.

De los 8 casos con DP umplio sólo 3 evolucionaron con insuficiencia cardíaca a partir del 50 dia de vida, requiriendo tratamiento médico habitual en restricción de volumen. de líquidos diuréticos y digitálicos

En los 8 niños con DP amplio se emplé indometacina en dosis de $0,2 \mathrm{mg}$ por kilo por dosis, oral, en 3 dosis calda 12 horas. En 6 de ellos hubo evidencias clínicus de cierre del DP en forma precoz (3 dias). En los otros 2 el cierre ductal fue más tardío.

Sólo 1 de los niños con DP amplio requirió tratamiento quirúrgico, teniendo und cvolución

Figura 3. 1.eociadiogralía de contraste grado I (arriba) y demostratción aor lográfica de un ductue pequeño (alsajo). sn el misno niño.

muy satisfinctoria.

Los restantes evolucionaron asintomáticos desde el punto de vistal cardioyascular.

\section{DISCUSION}

Nuestros hallazgos confiman el hecho que los elementos clínicos, radiográficos y electrocardiográlicos con frecuencia no son especiticos en e) diagnóstico preco $\%$ de la persistencia del ductus ел el nir̃o do pretérmino $13,16,21$. El aporte de la ecocardiografía Modo- $M$ en lo referente a relación $\mathrm{AI} / \mathrm{AO}$ es útil sólo en los DP amplios. andupe to especilica $0.14,20$. 
A pesar que el objeto del trabajo no fue realizar un análisis clínico detallado $y$ evolutivo de los pacientes, cabe destacar que sólo 1 de los 11 niños con DP aortográfico presentó soplo cardíaco durante las primeras 72 horas de vida.

La EC resulta ser un método simple, fidedig. no, inocuo y factible de realizar en la cuna del niño: de hecho, 15 de los prematuros estudiados fueron sometidos al examen en la Unidad de Neonatología, encontrándose incluso 7 de ellos conectados a respirador. El aprovechar el catéter arterial umbilical que se emplea rutinariamente como parte de la monitorización del prematuro con problema respiratorio para efectuar la EC, hace de ésta una técnica no invasiva.

La correlación aortográfica ecocardiográfica de contraste encontrada fue excelente en todos los casos. excepto en los casos 12 y 13 cuya aortografía fue negativa, pero cuyo $\mathrm{EC}$ fue tenuemente positiva. En ambos casos, e] catéter arterial se encontraba ubicado por bajo la 8 a vértebra torácica y probablemetite el Hypaque inyectado manualmente no logró contrastar un ductus contraido. En cambio. las microburbujas en el EC logran más fácilmente alcanzar la arteria pulmonar.

La busna cotrelación encontrada entre lo obtenido por EC y aortografía nos lleva a concluir que la EC. es método complementario de la cocardiografia Modo-M, de alto rendimiento, simple e inocuo, que permite un diagnôstico precoz de DP, to que conducirá a un manejo terapéutico también precoz en al recién nacido dé pretérmina.

Creemos que cl valor real de la puesta en marcha de esta técnica nos conducirá, además de hacer el diagnóstico precoz de DP, a valorar el real aporte de los datos clínicos, electrocardiográficos, radiológicos y ecocardiográficos Modo-M disponibles et nuestro medio y realizadus precozmente en el prematuro. Ello nos permitira en una primera ctapa, conocer la historia nalural del DP en el prematuro y así en el luturo poder predecir con rejativa exactilud cuáles y on qué momento estos ductus requirirán trataniento medicamentoso o quirúrgico para su cierre y tuáles cerrarán espontáneamente. De lograr lo expuesto, creemos que en un número importante de niños Hegaremos a prescindir de lécnicas invasivas, incluso de la $\mathrm{FC}$.

\section{RESUMEN}

Fl ductus persistente (DP) es más frecuente mientras menor es la edad gestacional del reciếr nacido, afectando adversamente su estado cardiopulmonar. Frecuentemente no existen suplos. otros signos clínicos son atípicos y el ecocardiograma Modo-M sólo da signos indirectos. En este trabajo demostramos li posibilidad de diagnosticar DP en el prematuro mediante un método simple de tcocardiografía de contraste (EC). El diagnóstico se cerfificó con aortografía ( $A O G$ ). En 16 prematuros menores de 72 horas de vida $y$ peso inferior a 2.000 granıos, se realizó EC a través de un catéter colocado en arteria umbilical entre la $4 a$ y 8 a vértebra dorsal se inyectó suero glucosado con registro ecocardiográfico simultáneo de arteria pulmonar (A.P.) y luego dortografúa. Según intersidad de ecos en AP, el EC se clasificó en grado I (ccos escasos) o grado Il (ecos densos). En la Aog, el tamaño del ductus se expresó según rélación diámetro ductal/diáme tro aorta descendente: DP amplio: relación mayor de 0,5 ; DP pequeño: relación menor de $0,5 . \mathrm{La}$ correlación ecocardiográfica-áortográfica fue cxcelente: 11 casos con DP aortográfico tenían FC positivo: 8 DP aortográfico amplio con EC grado 11: 3 DP aortográfico pequeño con EC grado l. Esta técnica de EC es sencilla de realizar y fidedigna para detectar precormente ol DP en prematuros, permitiendo predecir su tamaño y seleccionar los casos que requerirán tratamiento precoz.

\section{REFERENCIAS}

1. Bromn. E.R.: Increased risk of brondhopulmonary dysplasia in infants with patent ductus artcriosus. J. Padiatr. 95: 865, 1979.

2. Dudell, G.: Gersony. W.: Patent ductus arteriosus in neonates with sevure respiratory disedse. J. Podiatr. $104: 915,1984$.

3. Frituman, W.f'.: Patent ductus arterjosus in respiratory Distress Syndrome. Pediatr. Cardiol. 4 (Supp. Il): 3,1983 .

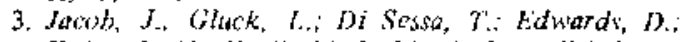
Kutorts, N.: Kurlinski. S.; Mertir, T.A.; Ariedman. W. F: The contribution of PDA in the neonate with svere RDS. J. P\&diatr. 96: 79. 1980.

4. Veal, $W_{. ;}$Bessinger, B.; Hunt, C.: Lucas, R.: Patent ductus arteriosus complicating respuratury disloss syndrome. J. Pediatr. 86:127, 1975.

5. Pickering, D.; Rigby, H.J..: Wilkinsum, A.R.: Incidence and clinical features of patent ductus arteriosus in promature infants with respiratory distress syndrome. Padial (a) (adiol, 4: (Suppl. II) 39,1983

6. Thibaut, D.W.: Emmanouilides. G.C.; Nelson, R.J.: lachman, R.S., Rosengart. R.M.: Oh, H.: Patent ductus artiriosus complicating the respiratory distrese to premature infants. J. Pediatr. 86: 120, 1975 .

7. Mofrath, R.I.; MeGuime's, G.A.; Way, G.l., The stlent duclus arteriosus. J. Pediatr. 93; 110, 1978.

8. Bath, R., Fisher, E., Raju, TN.K.; Valyasagar, D.: Pateat ductus arteriosus: Recent advances in diannosis and managemen1. Pedicte, Clin. North. AIt) 29: 1117,1982 . 
10. Baylen, B.G.; Mayer, R.A.; Kaplan, S.; Ringenburg, W.E.; Korfhogen, $J$ : The critically ill premature infant with patent ductus arteriosus and pulmonary disease: An echocardiographic assessment. J Pediatr. 86: 423, 1975.

11. Baylen, B.G.; Mayer, R.A.; Korfhagen, J, Benzing, G.; Bubb, M.E.; Xaplan, S.: Left ventricular per formance in the critically ill premature infant with patent ductus arteriosus and pulmonary diseass. C.irculation 55: 182, 1977.

12. Fisher, E.A.; Sepheri, B.; Barron, S.; Hastreiter, $A, R_{\text {.: }}$ Echocardiographic distolic flutter of the pulmonary valve in isolated patent ductus arteriosus, Chest, 81: 74, 1982.

13. Heitz, F.; Fouron, f.C.; Doeshurg. N.; Teasdate. F.; Chessexx, P.; Davignon, A.; Houde, L.: Value of sistolic time intervals in the dingnosis of large patent ductus arteriosus in fluid restricted and mechanically ventilated preterm infants. Pediatrics 74: $1069,1984$.

14. Hirschklan, M.J,; Di Sersa, T.G.i Higgins, C.B.; Friedman, W.F.: Jichocardiographic diagrosis: Pitfall in the premature infarit with a large patent duct arteriosuk. J, Pediatr. 92: 474, 1980.

15. Johnson, G.L.; Breart, G.i Genitz, M.; Brenner, J.;

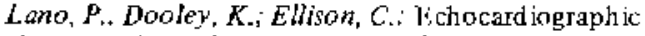
characteritics of prematures infant with patent ductus. Pediatrics 72:864, 1983

16. Johnson, G.L.; Nimala, S.D.; Cottrill, C.M.;
Johson, M.: Noonan, J.A.: Echocardiographic systolic time intervals in premature infants with patent ductus arteriosus. Pediatr. Cardiol. \&: 103 , 1980.

17. Silverman, N.H.; Lewis, A.B.; Heyman, M.A.; Rudolph, A.M. Echocard iographic assessment in ductus arteriosus shunt in premature infants. Circulation. 50: 821, 1974.

18. Gramiak, R.; Shoh, P.M.; Kramer, D.H.: Ultrasound cardiography contrast studies in anatomy and function. Radiology 92: 939, 1969.

19. Higgins, C.B.; Di Sessa, T.; Kirkparrick, S.E.; Ching, C.T. Edwards, D.K.; Friedman, W.F.: Kelly, M.J.; Kurlinsky. J.: Assessment of patent ductus arteriosus in preterm infants by single lateral film aortography. Radiology 135: $641,1980$.

20. Zednikava, M.; Baylen, B.G.; Yoshida, Y.; Emmanoulides, G.C.: Precordial contrast echocardiographic detection of patent ductus arteriosus in sunall premature infants. Pediatr. Cardiol. 2: 271.1982.

21. Allen, H.D.; Sahn, D.J.; Goldberg, H.J.: New serial contrast technique for assessment of left-to-tight shunting patent ductus arteriosus in the neonate. Am. J. Cadiol, 41: 288, 1978.

22. Sahn, D.J.; Allen, H.D.; George, W.i Mason, M.; Goldberg. S.J.: The utility of contrast echocardiographic techniques in the care of critically ill infants with cardiac and pulnonary disease. Circulation 56 : 959. 1977. 